\title{
The addictively trends of published research in medical journals: A mining approach
}

\author{
Alireza Atarodi ${ }^{1}$, Meisam Dastani ${ }^{2 *}$ and Ahmadreza Atarodi ${ }^{3}$ \\ ${ }^{1}$ Knowledge and information science, Department of Basic Medical Sciences, Paramedical College, Gonabad University of Medical Sciences, Gonabad, Iran \\ ${ }^{2}$ Knowledge and information science, vice chancellor for research and technology, Gonabad University of Medical Sciences, Gonabad, Iran \\ ${ }^{3}$ Research Committee, School of Medicine, Gonabad University of Medical Sciences, Gonabad, Iran
}

\begin{abstract}
Introduction: Medical researchers publish articles in medical Journals in different fields and topics in a trend that sometimes is not the way it should be and there is skewness to the left or right in topics being published in the said Journals. We are to find and warn to encourage the researchers and the men of responsible to make a better plan for Journal publications.
\end{abstract}

Methods: A simple survey in medical literature in books, Journals and different information sources was done. Then, text mining algorithms including vocabulary frequency and K-Means clustering were implemented using coding in the Python version-3 programming language on data.

Results: There was not a standard and planned style for article publishing in medical Journals and topic priority has not been in order and based on the need and necessity of the societies, For example the most observed categories in some medical Journals was on some topics mostly about "The education of medical students" and the term "student", "medical" education", learning and such so was more highlighted than the others.

Conclusion: Journals topics and the articles publishing should be controlled and the researches should also be directed to the topics being needed and topic priority for article research and publishing should be considered and a major concern to save time, costs and lives at the end.

\section{Introduction}

Human being needs knowledge and science to develop, grow and make the world as it should be and these knowledge and science should be disseminated in different information resources, one of which are Journals. The researches results are published and medical Journals are the base of publishing the results provided by researchers. What the researches research, do and publish is important and should be based on the needs, the aims and goals of the map way, mission and vision, put forward to reach the edge and find the target for solving the problems and challenges threating us. This is a trend needed to follow, and a trend is a pattern of gradual change in a condition, output, or process, or an average or general tendency of a series of data points to move in a certain direction over time, represented by a line or curve on a graph something special [1].

Since too many Journals are published each year and recent bibliometrics show that the number of published scientific papers has climbed by $8-9 \%$ each year over the past several decades. In the biomedical field alone for example, more than 1 million papers pour into the PubMed database each year and it is about two papers per minute. The number of scholarly articles published each year has grown steadily by about $3 \%$. Indeed, thirty years ago, Betty L. Siegel, President of Kennesaw College, observed, "There is enough scientific information written every year to keep a person busy reading day and night for 460 years. It is also interesting to note that while overall there is an average of 58.1 articles published per year in each medical journal, the titles now indexed in the Current List yield 71.3 articles per year per title [2].
Now, what subject and fields are covered by the medical Journals in the world? Do they publish the article titles based on the need of the societies and communities, are they said what to publish and what field has the vacancy to be filled by such kind of publications in the said medical Journals? These concerns are something we must be aware and anxious to know to make a correct plan for publication trend. The trend should be reformed, classified and evaluated. Medical education needs different subjects and is faced with different challenges to be solved, the number of publication per year/per researcher or per title is not the only way and aim in medical sciences areas. The researches should be planned and based on needs not on wants and wishes, since the old saying recite, wishes don't wash dishes. The collection of publications and filling the body of the Journals is out of date and not a good success today. Now with increasing the number of scientific articles in each field of science, identifying important scientific topics and researches is a special area of scientific necessity, then this research deals with the identifying of trends in published research in medical Journals by text mining approach. We aimed in this study identifying the most prioritized and important issues and topics in medical Journals

${ }^{*}$ Correspondence to: Meisam Dastani, Msc of knowledge and information science, vice chancellor for research and technology, Gonabad University of Medical Sciences, Gonabad, Iran; E-mail: meisam.dastani@gmail.com

Key words: data mining, medical journals, medical publications, text analysis, text mining

Received: March 04, 2020; Accepted: March 13, 2020; Published: March 19, 2020 
to be more considered and investigated to be a start for the Journals of medical sciences to cover all the needed and necessary topics for accepting and publishing throughout the scientific world.

\section{Materials and methods}

A survey was conducted first on the literatures related to the subjects, the most relevant literature and resources, books, and articles of the most relevant research topics, mostly the articles of the Journals in the same area and field for descriptive section of the research, however it is an applicational one in its type. This applicational study has analyzed the articles published over the past fifteen years using text mining and exploratory descriptive approach. The data of this study for some Journals publications has been extracted annually by direct referring to the website of medical science journals in bibtex format. Finally, all of these files have been merged into csv format using Jabref software. Then, text mining algorithms including vocabulary frequency and K-Means clustering were implemented using coding in the Python version-3 programming language on data. The necessity Tables and Figures were brought in the text. Some international and Iranian Journals were surveyed and studied and the needed results were extracted from them.

\section{Results}

In this study, the records were extracted from articles published and analyzed using text mining techniques. Different Journal showed different results for different topics and publication in some special area and topics. Scattering and dispersion of publishing in most of the Journals is observed that need to be reformed and prioritized.

Fontelo et.al found in their research that in 20-year review of publications only 30 countries (out of 235 total reviewed) were responsible for an average of $93.9 \%$ of all publications in PubMed. What might explain the few numbers of countries responsible for a majority of the world's literature? It is likely a consequence of country priorities, lower levels of funding, and the high cost of doing research, although labor costs may be lower [3]. Biomedical research is often not a top priority in developing countries. However, although the research output of developing counties may be relatively low, many important medical and public health interventions were developed in developing countries, such as life-saving interventions like oral rehydration therapy for diarrhea and vitamin A to reduce infant mortality [4]. Moreover, collaborative research may be indexed as publications from funding agencies or collaborators in developed countries. Other countries may also have fewer research and academic institutions. Then they recommend that developing countries could make important contributions to the medical literature, especially in the area of infectious diseases, and increasingly, non-communicable chronic diseases. Top research countries and research funding agencies may need to collaborate more with other countries or to select the topics the world need to be researched. Some of this may be occurring through the internationalization of clinical trials that are now done worldwide and increased student and fellowship training [5]. As it was said in top, some limitations limits and close the way for some countries and the researchers as well tending to some topics and priorities to conduct and publish and they then shift to the topics mostly accepted by Journals without any necessity and value of solving the problems and challenges the world is faced with and in need of practice.

Table 1 shows the 1 st 5 countries among 30 countries conducting different types of articles published in medical Journals in the world, that the USA ranks the first and China the next but with more publishing Meta-analysis than USA (4659 against 3256) in the year 2015 only.
Table 1. The first 5 top published countries in 2015

\begin{tabular}{|c|c|c|c|}
\hline The No. & Country in Year 2015 & All publication Types & The country Rank \\
\hline 1 & USA & 323047 & 1 \\
\hline 2 & China & 144853 & 2 \\
\hline 3 & UK & 88871 & 3 \\
\hline 4 & Germany & 68360 & 4 \\
\hline 5 & Japan & 58201 & 5 \\
\hline
\end{tabular}

Some other basic research results showed that in some other areas for example in scholarly journal publications on the topic of ecosystem services have substantially grown throughout the past decade, which is evidenced by an expanding world-wide research base. However, all the field is not covered and as the claim several challenges in the field of ecosystem services still remain, including conflicting approaches to ecosystem services terminology, classification schemes, research methods, and reporting requirements. They also assessed the development and disciplinary focus of ecosystem service research over the last decade using a meta-analysis of peer-reviewed journal publications. The journals of Shengtai Xuebao Acta Ecologica Sinica, Ecosystem Services, PLoS One, and Ecological Economics lead scholarly journals publishing ecosystem services-focused articles. The publications of the countries, however, were different in the said topic, as the European Union leads ecosystem services publications (42\%), while the United States is responsible for $30 \%$ of ecosystem services publications. This analysis is supplemented by a discussion on ecosystem services-focused research and policy to identify trends that encourage future study to resolve some of the current issues faced by the research community. This needs more cooperation and interaction to make the best use of it [6].

Some other researches and Journals have mostly focused on special topics only and mostly cover the education and teaching of medical students, since some academic centers have and publish a few number of Journals and then are forced to accept the general topics of all field and some special ones are observed and mostly viewed, one of the medical universities have no more than two medical Journals publishing articles of any topics, when the published articles in them is analyzed then some of the items or topics are more highlighted and repeated than others.

For example, some of the medical Journals in Iran were surveyed and their contents were analyzed through data mining approach and the results showed the mostly repeated words and terms drag out of the articles published. Then, the extracted terms or items were put in 3 clusters from $0-2$.

Silhouette criterion coefficient was 0.372 which evaluated 3 selected clusters as desirable in one Journal being analyzed. The results of implementing the Cummins clustering algorithm are shown in Table 2 along with the commonly used words of each cluster [7].

Table 2 shows that the zero cluster was related to the thematic axis of "clinical nursing education and students", cluster no. 1 was the "medical education of medical and academic students", and the cluster no. 2 was based on "the quality of education and teaching of students". The cloud of words being repeated in the articles published in medical Journals are also shown in fig. 1 below [7]:

The results in figurel shows that some categories formed a theme or phrase more highlighted than the others and that is "Education of Medical Students" even some others are also found but is much less than other themes merged of frequent words or term repeated in the articles published in medical Education Journals. 
Table 2. Topic clusters with important and commonly used words for each cluster

\begin{tabular}{|l|l|}
\hline Clusters & \multicolumn{1}{|c|}{ Mostly Repeated Words } \\
\hline Cluster-0 & $\begin{array}{l}\text { 'nursing', 'clinical', 'students', 'education', 'scores', 'effective', 'learning', 'used', } \\
\text { 'tested', 'means' }\end{array}$ \\
\hline Cluster-1 & $\begin{array}{l}\text { 'students', 'medical', 'academic', 'education', 'learning', 'university', 'scores', } \\
\text { 'courses', 'significant', 'sciences' }\end{array}$ \\
\hline Cluster-2 2 & $\begin{array}{l}\text { 'education', 'medical', 'factor', 'research', 'health', 'used', 'quality', 'university', } \\
\text { 'teaching', 'sciences', }\end{array}$ \\
\hline
\end{tabular}

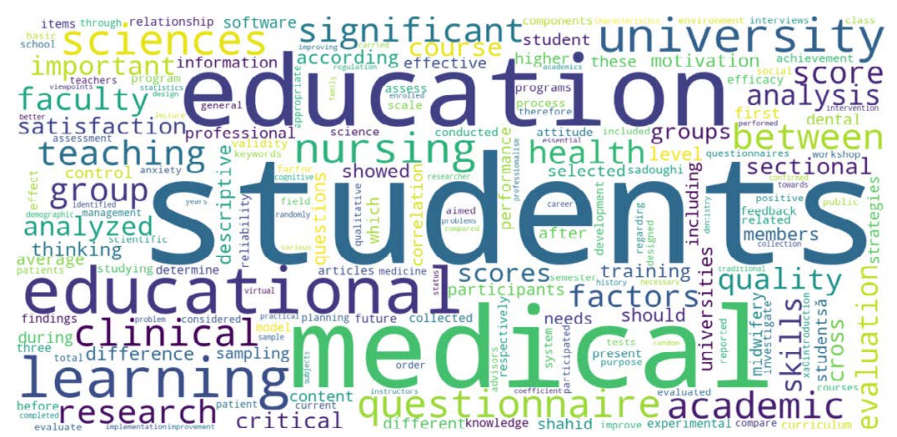

Figure 1. Frequently-words cloud in the articles published in medical Journals

When Muayyad Ahmad from the University of Jordan ask a question in virtual area of internet in 12th Dec, 2015 as the following: priority?"

"Which of the following topics you will choose as a research topic

Some answers were as the followings selecting from a list of topics being present by Muayyad Ahmad:

12th Dec, 2015

Miranda Yeoh

\section{SEAMEO RECSAM}

"It may be due to the culture around me that I choose alternative medicine. Here, several people do their best to take herbal medication, until it is absolutely necessary to take 'western medicine'. But herbal remedies are not yet so well researched. Thanks."

12th Dec, 2015

Laila Al daken

Sultan Qaboos University

Dear Professor Muayyad,

Greetings,

"I think the choice is not easy because there are many interesting and actually worthy topics among the above list. However, for me, I think any study about the AIDS and addictions among health care professionals will be valuable." Best luck,

12th Dec, 2015

\section{Ahmad Deeb}

King Abdullah International Medical Research Center

"In general I would focus on "emergence of novel infectious diseases" as research priority such as MERS and Ebola. From the list I would choose: and now may COVID-19 if was in the list od before.

Alzheimer's disease (new inventions still been reported and the hopes to find treatment are expanded)
Chronic pain (by targeting patients in low income countries who complained with this pain and they are not able to manage and cure it, despite the availability of advance pain management)

Cell phone use - dangerous? (it is still vague topic, need more exploration - the risks behind using cell phone (radio waves) - or - the positive and negative impact of using cell phone as a services on our life e.g. for life planning, work, reminders, use of social networks)

Alternative medicine (the misuse of alternative medicine or the potential impact of alternative medicine in some diseases)".

\section{Thanks}

12th Dec, 2015

\section{Abdulqadir J. Nashwan}

\section{Hamad Medical Corporation}

"I do believe that the selected topic should match the departmental, national, regional or international priorities, interest is not enough" [8].

But in some other places such as Canada with senior population increasing, there is greater demand for family physicians with enhanced skills in Care of the Elderly (COE). Since they have more problems and challenges with their future population of elderlies, they present and recommend different priorities in relation most with COE. Then, they are going to summarize the process used to determine the Priority Topics for the assessment of competence in COE, as they say. There has been a shift towards competency-based medical education (CBME) of residents. A core competency (CC) is a fundamental knowledge, ability or expertise in a subject area or skill-set within a specific field of care. Competencies relate to the skills, behaviors, and knowledge that are gained through training or practice, and explicitly define what a capable physician should be able to do to practice safely and effectively. The use of competencies, to guide curriculum and assessment in postgraduate medical education, is a significant model change from a focus on clinical knowledge and numerical ratings on In-training Evaluation Reports (ITERs). Professional competence is more than factual knowledge and ability to solve problems. It is defined by the ability to manage ambiguous problems, tolerate uncertainty, and make decisions with limited information. "Priority Topics" and "Key Features" are an approach to assessment which focuses on the most critical clinical elements and issues [9]. The said literature above present a new shift to some topics more needed in that society and area than repeated topics being published and taught in classrooms without any planned programs. According to their results, the identification of the Priority Topics was the first step in planning for the assessment of competence in COE for family physicians at the Enhanced Skills level. Now we can claim that Medical Journals need a priority assessment, too.

Noroozi, et al. in a research aimed to identify and prioritize the most important medical ethics issues for research in the Islamic Republic of Iran. They claim in their results that apart from universal moral standards, any society's culture, religion and behavior patterns have major impacts on ethical norms and bring different necessities and in this case the goals reflect the importance of research in medical ethics; limited resources mean that prioritizing research activities is extremely important. The researchers emphasize to determine the priorities of topics needed for research to be published in Journals and to fill the gaps. According to their states, after operationalizing the strategic plan in 2002, scientific production increased as exemplified by the number of articles published during 1990-2014 by Tehran University of Medical Sciences (315), Shahid Beheshti University of Medical Sciences (126) 
and Shiraz University of Medical Sciences (58) [10]. This shows the importance of topics priority for research and publication and it is then a necessity to make a plan for it, why it seems that in most limited studies have been conducted to identify the most important and prioritized medical topics and even in some more fields and disciplines of education in the world that should be reformed and changed.

Then, it seems that in most of the countries and in Iran for example, limited studies have been conducted to identify the most important and prioritized medical ethics topics; since this study has been conducted on medical ethics and some in Canada that request for the necessary research to be done for the Elderlies according to their future population or aging challenges [11].

Another research titled "Trends in Scientific Literature on Addiction to the Internet, Video Games, and Cell Phones from 2006 to 2010" was conducted to retrieve the scientific articles published on addiction to the Internet, video games, and cell phones and to analyze the pattern of publications in this area and claimed that most of the articles during the said years were published in some countries such as China $(n=67)$, The Unites States $(n=56)$, The United Kingdom $(n=47)$ and Taiwan $(n=33)$ and mostly in English language $((70.3 \%)$ in less than 5 Journal in the same countries and mostly read source was internet. They finally suggest changing the publication trend in technology ruling and some Journals totalitarian in publishing [12].

\section{Conclusion}

We reached to the point that prioritizing research activities is extremely important and necessary, the journals should also be prioritized by topics and make a map way to know what to accept and what to publish. From another standpoint, in order to support professional development and the advancement of research, designing a research road map for medical activities is required. Road mapping is a consensual process which identifies the best way to proceed [13]. Prioritizing research areas is the primary step for road mapping; it identifies a clear strategy for future investigations by addressing specific research questions and changing priorities [14,15]. Thus, interventions should arise from valid prioritization of problem [10]. The Journals of medical sciences are in contact with people and their living from or even before birth to the end or even after death with their qualitatively affects, then they should more care on their aims, publications and the way they go. The resources are limited, and the special researchers are not many and always and everywhere accessible, Journals publication fees are another challenge for the institutions and companies or academic centers and so many reasons that can be said. Then these all make us to think differently and find a way out of these problems and challenges and it seems that it is Topic Priority for article publishing and doing special researches based on need and necessity of the field and proposing from different centers in need of research practice to solve their problems. Anyway it means that in final that we have no much time and money not to have any program or mission and vision for a long enough time. When we found that in some journals the thematic clusters showed that the main theme was the word "education" or "medical student education" which was bolder and the rest of the findings revolved around the same theme and words and most of the articles topics have focused on education, missing some of the other requirements and topics or in ecosystem services and life-saving in developing countries. It should be indicated that all must and should know themselves responsible for publications to make their world much more pleasure and more in peace without any stress and anxiety. The world and mostly the researchers should cooperate in scientific writings, topic priority, developing culture, ethics, social capital, new discoveries and technology, inventions, controlling wars and unconventional weapons. We should write and do research to save life and try to find the area that has any problem to fill our body of the medical Journals with a human need for health since health makes wealth and this should be a belief and a hard belief. Since so many and uncounted concerns is against the human kind today and in some areas and mostly developing countries they have not any choice and a way out, requesting the world to help them and save, so many children are crying from the pains and being ill, drown in trouble, facing with very small and unimportant viruses but so dangerous and mortality rate for them, such as Covid-19, today in more than 30 countries waiting for a pleasure and saver message from around the world to be heard through all medias. Then what we write, what we research and tend to publish must and should be based on an aim, the aim that save, help, develop and grow security, health, social life, people grows with esteem and respect to help him for reaching to the level of self-actualization. This is the end and final goal of the human being, we think. What about you? But, this should be considered that topic priority must be based on Research Trends providing objective insights into scientific trends based on bibliometrics analyses since it is a worldwide growing demand for quality research performance measurement by researchers, funding bodies, academic and health centers and with a look on the world necessities and needs. Then, as Jafarian, et al. we also believe that priority setting in topics for publishing should be considered important and the effect size of each issue on the intended field is also important in priority setting [16]. And at the end underatanding Trend analysis is a process used in technical analysis trying to predict the future stock price movements based on recently observed trend data and information available and it is based on the idea that what has happened for the research outcomes in the past provide all researchers and men of the field an idea of what will happen in the forthcoming days. Trend analysis helps the analyst to make a proper comparison between the two or more Journals or publishers over a period of time and can also show them where they stand before/now and where should be in the future and it helps to understand the strength or weakness of themselves in comparison with others and the needs and world priority in publishing. Aimless publishing is not the aim and use.

\section{References}

1. Businessdictionary (BD) (2020) What is trend? Definition and meaningBusinessDictionary.com. www.businessdictionary.com. Definition trend.

2. Chi Y (2013) Global trends in medical journal publishing. J Korean Med Sci 28: 11201121. [Crossref]

3. Fontelo P, Liu F (2018) A review of recent publication trends from top publishing countries. Syst Rev 7: 147.

4. National Research Council (2006) The fundamental role of science and technology in international development: an imperative for the U.S. Agency for international development. Washington, DC: The National Academies Press.

5. Wagner CS, Park HW, Leydesdorff L (2015) The continuing growth of global cooperation networks in research: a conundrum for national governments. PLoS One 10: e0131816. [Crossref]

6. McDonough K, Hutchinson S, Moor T, Hutchinson SJM (2017) Analysis of publication trends in ecosystem services research. Ecosystem Services 25: 82-88.

7. Dastani M, Atarodi AR, Erfanpour S (2019) A study of published Research Trends in Iranian Journal of Medical Education. A written research plan, Research \& Technology vice presidency, Gonabad Uni. of Medical Sciences.

8. Muayyad Ahmad (2015) University of Jordan. Which of the following topics you will choose as a research topic priority.

9. Lesley AC, Chris CF, Tim A, Tatjana L, Marcel ASF, et al. (2018) Identifying the priority topics for the assessment of competence in care of the elderly. Can Geriatr $J$ 21: 6-13. [Crossref]

10. Noroozi M, Larijani B, Nedjat S, Aramesh K, Salari P (2019) Priority setting for research in the field of medical ethics in the Islamic Republic of Iran: a Delphi study. East Mediterr Health $J$. 
11. Bagheri A (2011) Iranian medical ethics priorities: the results of a national study. Iran J Med Ethics Hist Med 4: 39-48.

12. Carbonell X, Guardiola E, Fuster H, Gil F, Panova T (2016) Trends in scientific literature on addiction to the internet, video games, and cell phones from 2006 to 2010. Int J Prev Med 7: 63. [Crossref]

13. Ma T, Liu S, Nakamori Y (2006) Roadmapping as a way of knowledge management for supporting scientific research in academia. Syst Res 23: 743-755.
14. McGregor S, Henderson KJ, Kaldor JM (2014) How are health research priorities set in low and middle income countries? A systematic review of published reports. PLoS ONE 9: e108787. [Crossref]

15. Chalmers I, Glasziou P (2009) Avoidable waste in the production and reporting of research evidence. Lancet 374: 86-89. [Crossref]

16. Jafarian A, Parsapoor A, Hajtarkhani A, Asghari F, Imami Razavi SH, et al. (2009) A survey on the complaints registered in medical council organization of Tehran. Iran $J$ Med Ethics Hist Med 2: 67-74.

Copyright: (C2020 Atarodi A. This is an open-access article distributed under the terms of the Creative Commons Attribution License, which permits unrestricted use, distribution, and reproduction in any medium, provided the original author and source are credited. 